\title{
Astroglial D-serine is the endogenous co-agonist at the presynaptic NMDA receptor in rat entorhinal cortex
}

\author{
Alex M. Lench, Peter V. Massey, Loredano Pollegioni, Gavin L. Woodhall and Roland S.G. Jones
}

\section{Introduction}

Transmitter release at central synapses and, hence, the strength of synaptic transmission is controlled on a momentto-moment basis by activation of auto or heteroreceptors on presynaptic nerve terminals. At glutamate synapses, presynaptic NMDA receptors (preNMDArs) exert a tonic facilitatory effect on glutamate release and, thus, mediate an instantaneous form of synaptic plasticity. We first demonstrated this functional facilitatory effect at synapses in the rat medial entorhinal cortex (EC; Berretta and Jones, 1996a) and subsequently showed that it was mediated via $\mathrm{Ca}^{2+}$-entry via the receptor ionophore (probably di-heteromeric GluN1-GluN2B receptors) and could also contribute to activity-dependent facilitation (Woodhall et al., 2001 and Chamberlain et al., 2008). It is now apparent that preNMDArs are expressed at many sites throughout the CNS, and a widespread role of the receptors in control of transmitter release is likely (see Duguid and Smart, 2009). In particular, attention has increasingly focussed on a role in long-term synaptic plasticity of excitatory transmission (Humeau et al., 2003, Sjostrom et al., 2003, Samson and Pare, 2005, Corlew et al., 2007, Yang et al., 2008, Rodríguez-Moreno et al., 2010 and Larsen et al., 2011). Our observation that preNMDAr are highly mobile in the presynaptic terminal membrane in the EC (Yang et al., 2008) could indicate an important role in metaplasticity as well. Enhanced expression or sensitivity of the preNMDAr may be involved in pathological plasticity associated with epilepsy (Steffens et al., 2005 and Yang et al., 2006).

NMDAr are modulated by a number of intrinsic regulatory mechanisms. In addition to partial relief from voltagedependent $\mathrm{Mg}^{2+}$-blockade, NMDAr activation requires binding of two agonists; the glutamate binding site is located on the GluN2 subunit and a co-agonist binding site resides on GluN1 subunits. Both glycine and D-serine are available as physiological ligands for the co-agonist site. Although glycine has generally been considered to be the endogenous co-agonist, recent studies have indicated that D-serine is more likely to be the activator of the binding site at the postsynaptic NMDAr (postNMDAr), and that glycine is maintained at concentrations well below its affinity for the binding site by high-affinity transporters in the synaptic cleft (Fossat et al., 2012 and Papouin et al., 2012). The source of the endogenous synaptic D-serine may well be adjacent astrocytes (Henneberger et al., 2010 and Fossat et al., 2012), although a neuronal origin has not been ruled out (e.g. Wolosker et al., 1999, Kartvelishvily et al., 2006 and Rosenberg et al., 2010).

The EC plays a crucial role in declarative and spatial memory storage (e.g. Eichenbaum, 2001, Squire et al., 2004 and Witter and Moser, 2006) and in limbic seizure generation and propagation in temporal lobe epilepsy (see Jones and Woodhall, 2005). To help understand the role of the preNMDAr in synaptic function in the EC we have investigated whether they are subject to regulation by endogenous glycine or D-serine, and whether this regulation may be necessary for tonic facilitation of spontaneous glutamate release. Li and Han (2007) previously showed that an antagonist at the NMDAr co-agonist site was able to reduce the spontaneous release of glutamate in visual cortex, an effect that was reversed by addition of glycine suggesting that endogenous activation of the co-agonist-binding site regulated tonic activation of preNMDArs in this area. In the present study we show that preNMDArs in the EC undergo a similar endogenous modification, that the endogenous agonist responsible for this is D-serine not glycine, and that the source of the D-serine is likely to be astroglial cells.

\section{Materials and methods}

\subsection{Ethics statement}

All experiments were performed in accordance with the U.K. Animals (Scientific Procedures) Act 1986, European Communities Council Directive 1986 (86/609/EEC) and the University of Bath ethical review document, which requires that the number of animals used is kept to a minimum, and every precaution was taken to reduce suffering 
and stress. At this institution, all research work involving use of animal tissue requires submission of a consideration of ethical implications by the principal investigator. This is reviewed by a second investigator, external to the research group, and by the Head of Department, and requires signatory approval from both before being reviewed by a Departmental Research Ethics Officer (DREO). The DREO discusses any issues raised with the investigator and submits a report to the University Ethics Committee detailing the ethical implications of all research within the Department. This ensures that the ethical implications of the research have been considered and that there is a process in place for managing any ethical issues.

\subsection{Slice preparation}

Brain slices were prepared (Jones and Heinemann, 1988) from juvenile Wistar rats (50-100 g; P28-38). They were decapitated following cervical dislocation and the brain removed and submerged in oxygenated artificial cerebrospinal fluid (aCSF; see below for composition) at $4{ }^{\circ} \mathrm{C}$. Combined EC-hippocampal brain slices ( $350 \mu \mathrm{m}$ thick) were cut at $4{ }^{\circ} \mathrm{C}$ using a Campden Vibroslice and subsequently stored in oxygenated aCSF at room temperature. Slices were left to recover for $1 \mathrm{~h}$ before being used for electrophysiological recording. The aCSF contained (in $\mathrm{mM}$ ) $\mathrm{NaCl}$ (126), $\mathrm{KCl}$ (4), $\mathrm{MgSO}_{4}$ (1.25), $\mathrm{NaH}_{2} \mathrm{PO}_{4}(1.4), \mathrm{NaHCO}_{3}(24), \mathrm{CaCl}_{2}(2)$, d-glucose (10), ascorbic acid (0.57), sodium pyruvate (5) and creatinine monohydrate (5). To increase neuronal survival and viability, ketamine (4 $\mu \mathrm{M})$, indomethacin $(45 \mu \mathrm{M})$, aminoguanidine $(25 \mu \mathrm{M})$, and Coomassie Brilliant Blue (250 nM) were included in the cutting solution, and the antioxidants, n-acetyl-I-cysteine $(2 \mu \mathrm{M})$ and uric acid $(100 \mu \mathrm{M})$, added to both cutting and storage solutions. We have established that the use of the additives facilitates production robust and viable slices, but does not affect the pharmacology of glutamate transmission.

\subsection{Whole-cell patch clamp recordings}

After a period of recovery at room temperature, slices were transferred to a chamber on an Olympus BX50WI microscope perfused $\left(2 \mathrm{ml} / \mathrm{min}\right.$ ) with oxygenated aCSF at $31-32^{\circ} \mathrm{C}$, where they were allowed to equilibrate before recording commenced. Individual neurones were visualized using DIC optics and an infrared video camera.

Whole cell patch clamp recordings were made with an Axopatch 200A amplifier using pipettes pulled from borosilicate glass on a Flaming-Brown microelectrode puller. AMPA receptor mediated spontaneous excitatory post synaptic currents (SEPSCS) were recorded using a Cs-gluconate based solution intracellular solution containing (in $\mathrm{mM}$ ) d-gluconate (100), HEPES (40), QX-314 (1), EGTA (0.6), NaCl (2), Mg-gluconate (5), TEA-Cl (5), phosphocreatine (10), ATP-Na (4), GTP-Na (0.3) and MK801 (5 mM). The solution was adjusted to 275-290 mOsm by dilution and pH 7.3 with $\mathrm{CsOH}$. MK-801 was included in the patch pipettes to allow us to record AMPA-receptor mediated responses in isolation and to monitor activity at preNMDArs uncontaminated by postsynaptic receptor effects. We developed and validated this approach (Berretta and Jones, 1996b, Woodhall et al., 2001, Yang et al., 2006 and Yang et al., 2008), and others have used it successfully to block postNMDArs in recorded neurones (Sjostrom et al., 2003, Samson and Pare, 2005, Bender et al., 2006, Jourdain et al., 2007, Li and Han, 2007 and Brasier and Feldman, 2008).

EPSCs were recorded at a holding potential of $-60 \mathrm{mV}$. Signals were filtered at $2 \mathrm{kHz}$, digitised at $50 \mathrm{kHz}$, and stored using AxoScope software. Series resistance compensation was not employed, but access resistance was monitored at 5 -min intervals throughout recording and cells where it varied by greater than $15 \%$ were excluded from analysis. Input resistances for the neurones recorded in these studies were of the order 500-570 $\mathrm{M} \Omega$. Data recording commenced 10-15 min after gaining whole cell access and then continued for at least 15 min during control and each drug treatment condition. Miniature EPSCs (mEPSCs) were isolated using tetrodotoxin (TTX; $1 \mu \mathrm{M})$. EPSCs were analysed offline over a stable 5-min period of recording where spontaneous events were detected using a thresholdcrossing algorithm with Minianalysis software (Synaptosoft, Decatur).

The average frequency, calculated across a 5-min epoch, was compared before and after drug application. To compare amplitudes of spontaneous currents we determined median values for each neurone in the same 5-min period as these better reflect the population distributions (normal distribution with a slight skew towards larger amplitude events) than arithmetical means. Median values were then averaged in the neuronal populations for 
comparative illustrative purposes. We have used this approach in a previous study to characterize IPSCs in the same neuronal population (Woodhall et al., 2005). Event kinetics were compared via arithmetical means. Statistical comparison of drug effects on mean frequency and kinetics and mean median amplitudes within cells employed a two-tailed paired t-test. In experiments involving matched slices with and without prior enzyme treatment, comparison between neurones was assessed using a two-way ANOVA. In some experiments involving pooled data, we additionally compared cumulative probability distributions of interevent intervals using a Kolmogorov-Smirnov test.

\subsection{Enzymatic depletion of endogenous D-serine and glycine}

To determine whether glycine or D-serine is the endogenous activator of the co-agonist site we depleted one or other of the amino acids from the slices by preincubation with selective enzyme scavengers. An yeast d-amino acid oxidase (DAAO), was used to degrade D-serine, whereas a bacterial glycine oxidase (GO) was used to target glycine. Recombinant Rhodotorula gracilis DAAO (RgDAAO) and Bacillus subtilis glycine oxidase GO (BsGO) were overexpressed in Escherichia coli cells and then purified as described previously ( Fantinato et al., 2001 and Job et al., 2002). In experiments where they were employed, slices were incubated with enzymes at room temperature in the recording chamber for at least $45 \mathrm{~min}$ prior to recording, and then continuously during the recording period at $32{ }^{\circ} \mathrm{C}$. Matched slices in normal buffer were treated in the same way and alternated with the enzyme treated slices. For each experiment a single neurone was recorded from an enzyme treated slice, and a second recording was made in an untreated slice from the same animal on that day, so that neurones were matched in that animal. The order of recording (treated or untreated) was varied from animal to animal.

\subsection{Source of D-serine in EC}

Previous studies have suggested that a principal source of D-serine in slices is via release from astroglial cells (Kartvelishvily et al., 2006, Miya et al., 2008 and Rosenberg et al., 2010). The metabolic status of astroglial cells is compromised by sodium fluoroacetate (NFAc). Accordingly, we have determined the effect of NFAc on spontaneous release of glutamate in EC. In experiments where this was tested, slices were incubated with NFAc in the recording chamber for at least $35 \mathrm{~min}$ prior to recording, and also continuously during the recording period. Matched slices in normal buffer were treated in the same way and alternated with the NFAc treated slices.

\subsection{Materials}

Salts used in preparation of aCSF were purchased from Merck/BDH or Fisher Scientific (UK). Indomethacin, aminoguanidine, $n$-acetyl-I-cysteine, uric acid, Coomassie Brilliant Blue, NFAc and agents used in the preparation of the patch pipette solution, apart from QX-314 (Tocris, UK), were purchased from Sigma (UK). Ketamine was supplied by Fort Dodge Animal Health Ltd (Southampton, UK). Drugs were generally stored as frozen, concentrated stock solutions in distilled water and applied by dilution in the aCSF perfusion immediately before use. The following drugs were supplied by Tocris UK or Ascent Scientific UK: 2-AP5 (D-2-amino-5-phosphonopentanoic acid), DCKA (5,7dichlorokynurenic acid), CPPG ((RS)- $\alpha$-Cyclopropyl-4-phosphonophenylglycine), MCPG ((RS)- $\alpha$-Methyl-4carboxyphenyl-glycine), MK801, glycine, suramin hexasodium salt, CGS15943, D-serine and TTX. UBP 141 was a kind gift from Professor David Jane, University of Bristol.

\section{Results}

The experiments were performed on neurones in layer $V$ of the medial EC. Although not specifically identified by dye injection, all the selected neurones had identifiable pyramidal cell morphology when viewed with DIC. We stress that all recordings were of AMPA-receptor mediated s/mEPSCs with postNMDArs blocked in the recorded neurone by intracellular dialysis with MK801.

\subsection{Effects of D-serine and glycine}

The effects of exogenously applied glycine $(100 \mu \mathrm{M})$ were tested on 4 neurones and the effects are summarised in Fig. 1A. The mean frequency of mEPSCs in these neurones was $0.75 \pm 0.13 \mathrm{~Hz}$ in control conditions. This was similar $(0.73 \pm 0.12 \mathrm{~Hz} ;-4 \%)$ in the presence of glycine (Fig. $1 \mathrm{~A})$ and there was no significant difference when assessed with 
a paired t-test. We also compared cumulative probability distributions of interevent interval, and KS analysis showed no significant shift in distribution in the presence of glycine reflecting the lack of change in frequency. mEPSCs had a mean median amplitude of $6.4 \pm 0.2 \mathrm{pA}$ in control and this was not significantly altered during perfusion with glycine $(6.0 \pm 0.2 \mathrm{pA})$. Additionally, neither sEPSC rise $(4.7 \pm 0.8 \mathrm{~ms} \vee 5.3 \pm 0.3 \mathrm{~ms})$ nor decay time $(6.3 \pm 1.2 \mathrm{~ms} \vee 6.3 \pm 0.3$ $\mathrm{ms}$ ) changed during perfusion with the amino acid.

A
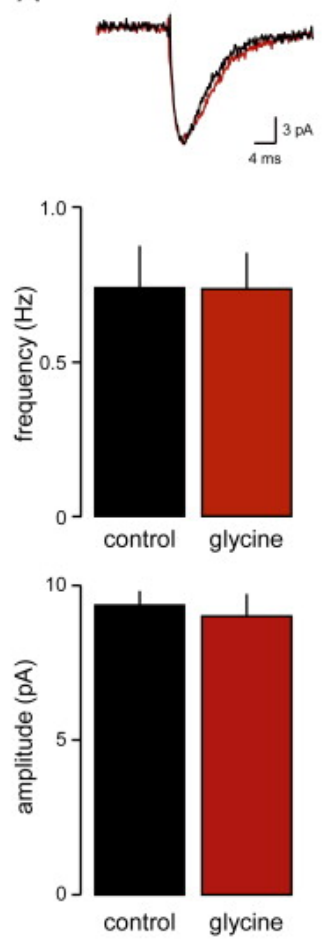

B
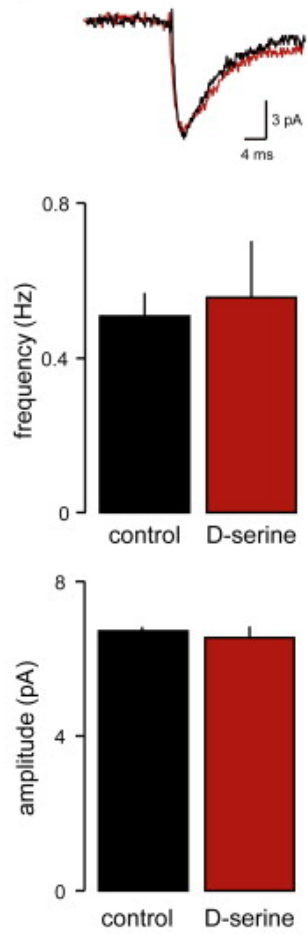

Fig. 1. Effect of D-serine and glycine on sEPSCs in EC neurones. A. The traces show averaged mEPScs in the presence and absence of glycine in a single layer $\mathrm{V}$ neurone in the EC. The addition of glycine had little effect on the excitatory events. Pooled data from 4 neurones in the bar graphs show that neither mean frequency nor amplitude nor was significantly altered. B. Studies with D-serine produced very similar results. A slight increase in mean frequency was not significant.

Similar studies were performed with D-serine (100 $\mu \mathrm{M} ; \mathrm{n}=4$; Fig. 1B). As with glycine, there was no significant change in mean frequency of mEPSCs $(0.51 \pm 0.06 \mathrm{~Hz} \vee 0.56 \pm 0.15 \mathrm{~Hz} ;+11 \%)$ during perfusion with the amino acid when assessed with a paired t-test. However, there was a small but significant shift to the left of the cumulative probability distribution of events when the data were analysed as interevent interval distributions (not shown) and compared with a Kolmogorov-Smirnov test $(P<0.05)$. This change was not pronounced but was the only significant change detected. Mean median amplitude $(6.7 \pm 0.1 \mathrm{pA} \vee 6.5 \pm 0.3 \mathrm{pA})$, mean rise $(4.3 \pm 0.3 \mathrm{~ms} \vee 4.5 \pm 0.3 \mathrm{~ms})$ and mean decay time ( $5.5 \pm 0.3 \mathrm{~ms} \vee 6.5 \pm 0.3 \mathrm{~ms}$ ) were unaltered by $\mathrm{D}$-serine.

\subsection{The preNMDAR co-agonist binding site is tonically activated}

The failure of either glycine or D-serine to substantially increase the release of glutamate may suggest that the coagonist binding site of the preNMDAr is tonically activated at a level close to saturation in the EC. If this is the case then blocking the site should result in a decrease in release. To investigate this we examined the effect of DCKA, a co-agonist binding site antagonist. Although postNMDArs were blocked in the recorded neurones by intracellular MK801, these experiments $(n=4)$ were again conducted on mEPSCs to avoid any complicating effects of network activity via non-recorded neurones in which the postNMDArs were not blocked. The results are shown in Fig. $2 \mathrm{~A}$. DCKA elicited a significant decrease in mEPSC frequency to around $60 \%$ of the control level. This effect was not accompanied by any change in mean amplitude $(8.37 \pm 0.5 \vee 7.8 \pm 0.4 \mathrm{pA})$, rise time $(4.8 \pm 0.3 \vee 4.8 \pm 0.4 \mathrm{~ms})$ or decay time $(5.8 \pm 0.5 \vee 6.0 \pm 0.7 \mathrm{~ms})$, and this lack of effect on mEPSCs is shown by the averaged events and the lack of change in amplitude distribution in Fig. 2A. Overall, these effects indicate that DCKA acts presynaptically to reduce the probability of glutamate release. 

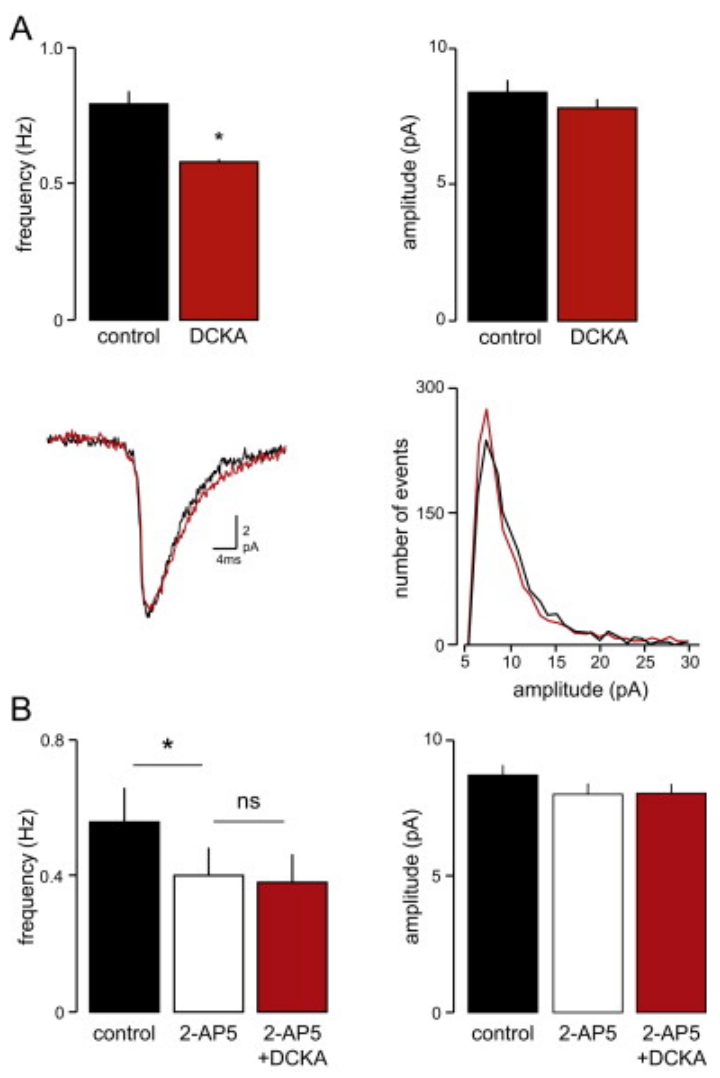

Fig. 2. A co-agonist site antagonist reduces spontaneous glutamate release. A. Application of the co-agonist binding site antagonist, DCKA ( $n=$ 5), produced a robust decrease in mEPSC frequency, but was without effect on event amplitude. The averaged traces from one neurone show no change in event amplitude or kinetics, and the lack of change in amplitude is also reflected in the frequency distributions of pooled data. $B$. The competitive glutamate binding site antagonist, 2-AP5 induced a similar decrease in mEPSC frequency to that seen with DCKA, and also occluded the effect of the latter. In this and subsequent figures, ${ }^{*}=P<0.05$ and ' $n s^{\prime}$ denotes not significant.

To confirm that the effect of DCKA was likely to be mediated via an action at preNMDAr we examined its effect in the presence of the competitive NMDAr antagonist, 2-AP5 ( $n=5$; Fig. 2B). In accordance with our previous findings ( Berretta and Jones, 1996a and Woodhall et al., 2001), 2-AP5 induced a significant decrease in mEPSC frequency (0.47 $\pm 0.04 \vee 0.29 \pm 0.05 \mathrm{~Hz})$ without change in amplitude $(8.0 \pm 0.8 \vee 7.6 \pm 0.7 \mathrm{pA})$. Subsequent perfusion with DCKA in the presence of 2-AP5 now failed to induce any further change in frequency. Thus, the occlusion of the effects of DCKA by the competitive receptor antagonist suggests that it is acting at preNMDArs and blocking the tonic facilitation of release enabled by binding of endogenous glutamate and a co-agonist.

\subsection{Glycine does not endogenously activate the co-agonist binding site}

Having determined that the co-agonist binding site of the preNMDAr was tonically and endogenously activated in our slices, we then attempted to identify the endogenous ligand responsible. The small, but significant, decrease in interevent interval induced by D-serine could suggest that the D-serine is the preferred ligand for the co-agonist binding site. To look at this further we selectively depleted the candidate ligands via enzymatic breakdown. This approach has been successfully used to look at endogenous ligands for the postNMDAr (Fossat et al., 2012 and Papouin et al., 2012). In the first set of experiments we examined the effect of degrading endogenous glycine with BsGO. We compared the characteristics of mEPSCs in 6 neurones in untreated brain slices to matched neurones in brain slices incubated with BsGO for 45 min.

Fig. 3 illustrates the results of these experiments. The frequency of mEPSCs $(0.41 \pm 0.13 \mathrm{~Hz})$ in slices preincubated with BsGO $(n=6)$ was not significantly different to that in matched slices without enzyme present $(0.33 \pm 0.04 \mathrm{~Hz})$. Likewise, the mean amplitudes ( $7.7 \pm 0.4 \vee 7.9 \pm 0.9 \mathrm{pA}$ ), amplitude distribution ( Fig. 3), and kinetics in treated and untreated slices (rise time $4.4 \pm 0.4 \vee 4.3 \pm 0.4 \mathrm{~ms}$; decay time $7.1 \pm 0.8 \vee 7.2 \pm 0.7 \mathrm{~ms}$ ) were not significantly affected by the enzyme treatment. These data clearly suggest that endogenous glycine is unlikely to be tonically activating the binding site on the preNMDAr. To confirm that the site was still being activated we determined the effect of 
DCKA in both naïve and enzyme treated slices. In both cases, the co-agonist site antagonist, as described above, reduced the frequency of mEPSCs ( Fig. 3) without altering either amplitude distribution ( Fig. 3) or kinetics (data not shown).
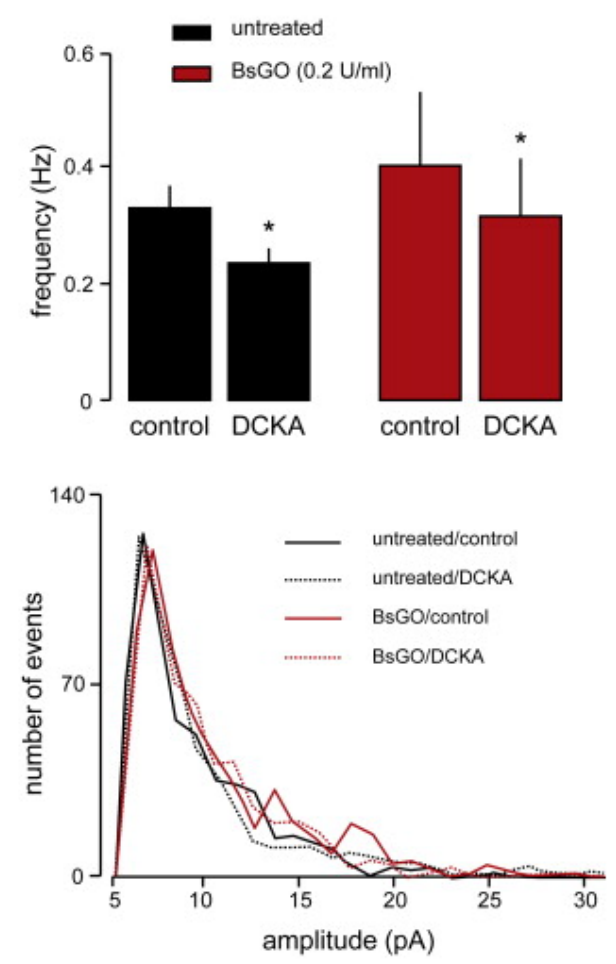

Fig. 3. Depletion of endogenous glycine does not affect spontaneous glutamate release. Comparison of mEPSC frequencies with and without pre-incubation with BsGO showed no significant difference between matched control slices and slices incubated with the enzyme ( $\mathrm{n}=6$ in each case). In the naïve slices, as noted in Fig. 2, DCKA elicited a significant reduction in mEPSC frequency, and this effect was very similar in the enzyme-treated slices. There were no differences in event amplitudes in any of the experimental situations and this is illustrated by the overlapping frequency distributions.

\subsection{D-serine endogenously activates the co-agonist site}

We next examined the effect of removal of endogenous D-serine from our slices by incubation with the yeast enzyme, RgDAAO, which specifically degrades D-serine (Fig. 4). The mean frequency of mEPSCs in 6 neurones in untreated slices was $0.65 \pm 0.13 \mathrm{~Hz}$. However, in 6 neurones in slices incubated with RgDAAO, mEPSC frequency was lowered to $0.38 \pm 0.03 \mathrm{~Hz}$. This substantial decline in frequency was not accompanied by differences in mean event amplitude $(7.8 \pm 0.5 \mathrm{pA} \vee 7.3 \pm 0.3 \mathrm{pA}$ ), kinetics (rise time $4.7 \pm 0.3 \vee 5.1 \pm 0.3 \mathrm{~ms}$; decay time $6.0 \pm 0.4 \vee 5.9 \pm 0.7 \mathrm{~ms}$ ) or amplitude distribution ( Fig. 4). As in the glycine experiments, we also studied the effects of DCKA. In naïve slices DCKA reduced the frequency of mEPSCs without change in amplitude or kinetics, as described above. However, pretreatment with RgDAAO occluded the effects of DCKA, and the co-agonist site antagonist caused no further reduction in frequency and no change in amplitude distribution ( Fig. 4). These results indicate that D-serine is likely to be the endogenous co-agonist at the preNMDAr, and that glycine, at least in the conditions pertaining to our slices, may play little role in this regard. 

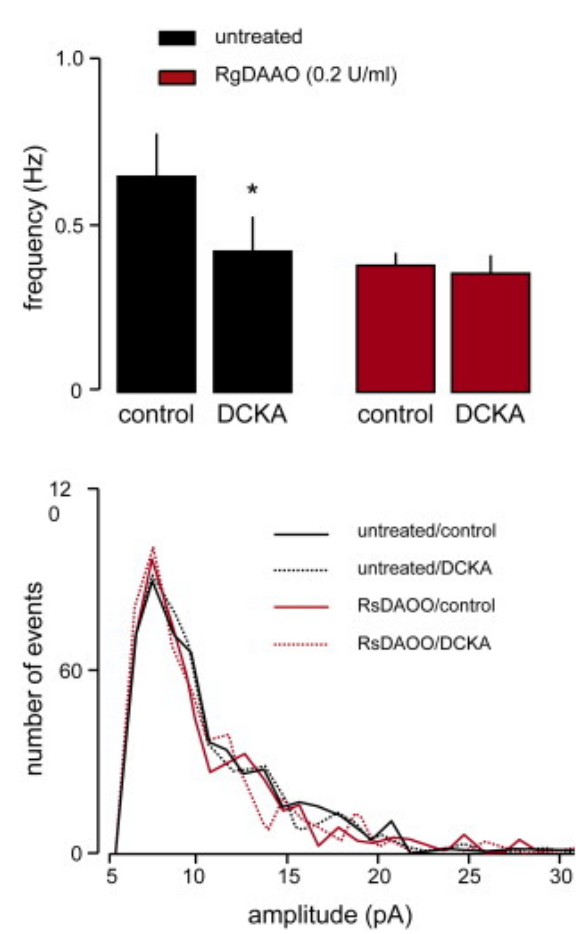

Fig. 4. Depletion of endogenous D-serine reduces spontaneous glutamate release. Pre-incubation of slices with the d-amino acid oxidase, RgDAAO to degrade endogenous D-serine resulted in a significant decrease in mEPSC frequency in comparison to matched untreated slices ( $\mathrm{n}$ $=6$ in each case). This reduction was similar to that seen with the co-agonist binding site antagonist, DCKA, and the effect of the latter was occluded by pre-incubation with the enzyme. Despite the change in frequency, event amplitudes were unaltered, exemplified by the overlapping frequency distributions of amplitudes from pooled data.

\subsection{Astrocytes may be the source of endogenous D-serine}

Previous studies have shown that D-serine is the likely to be the endogenous co-agonist at subsynaptic postNMDAr in cortex (Fossat et al., 2012 and Papouin et al., 2012) and that astroglia may be the source of the D-serine. We have looked at the possibility that astroglial D-serine may be the endogenous activator of preNMDArs in the EC. To this end we compared mEPSCs in neurones with and without preincubation with the glia-specific metabolic inhibitor, NFAc (Hassel et al., 1997, Hulsmann et al., 2003, Andersson et al., 2007 and Okada-Ogawa et al., 2009). The results are summarized in Fig. 5A. In 6 neurones recorded in naïve slices, mEPSC frequency was $0.66 \pm 0.13 \mathrm{~Hz}$ and mean amplitude was $7.0 \pm 0.7 \mathrm{pA}$. In matched recordings from slices pre-incubated with NFAc $(n=6)$, mEPSC frequency was much lower at $0.35 \pm 0.03 \mathrm{~Hz}$, but there was no change in mean amplitude ( $7.1 \pm 0.5 \mathrm{pA})$. The amplitude distribution of mEPSCs was also unaltered and there were no differences in either rise or decay times in the two groups (not shown). We also tested the effects of the co-agonist site antagonist in the presence or absence of NFAc ( Fig. 5A). In the untreated slices DCKA reduced the frequency of mEPSCs, as noted above. However, when tested in NFAc-treated slices, DCKA had little further effect on mEPSC frequency. Comparison of the effect of DCKA in the two sets of slices showed a trend towards a bigger reduction in frequency when combined with NFAc, but this was not significant. 

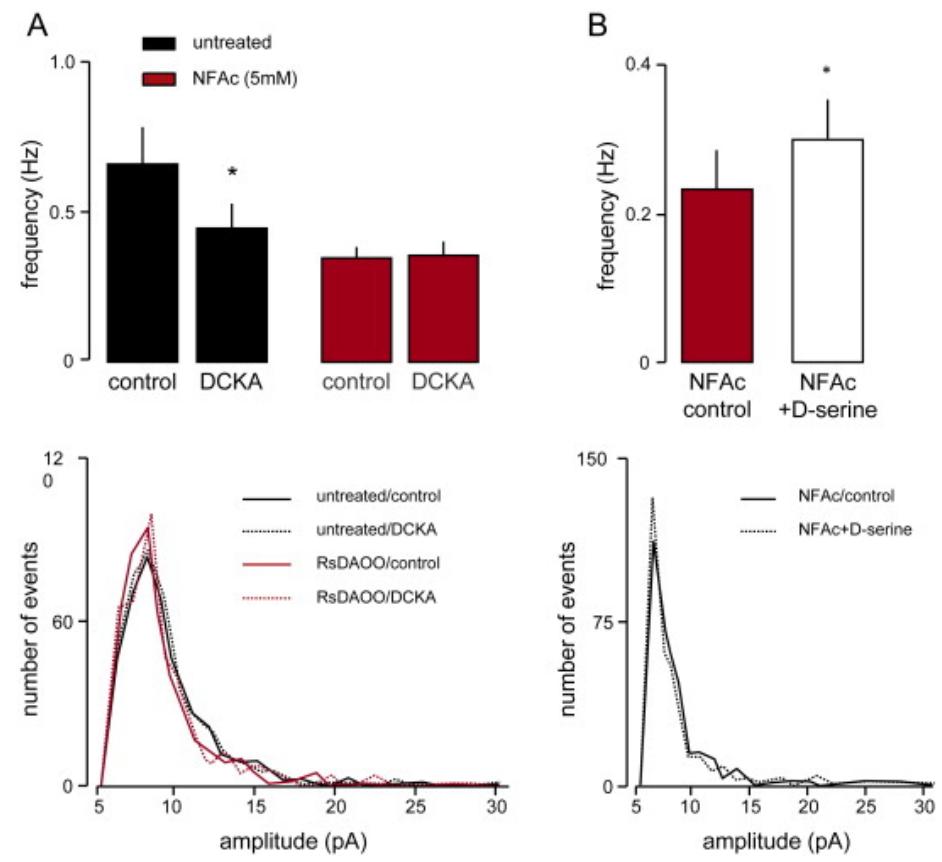

Fig. 5. Compromising astroglial function reduces spontaneous glutamate release. A. In slices pre-incubated with NFAc $(n=6)$, mEPSC frequency was substantially reduced compared to neurones in matched control slices $(n=6)$, and this effect was similar to that seen with DCKA and RgDAAO ( Fig. 2 and Fig. 4). However, the effect of DCKA was occluded by pre-incubation with NFAc. Amplitude distributions were unaffected by any of the experimental treatments. B. The reduction in mEPSC frequency seen with NFAc was partially rescued by application of D-serine. Thus, in contrast to the situation in untreated slices (Fig. 1), perfusion with D-serine increased the frequency of mEPSCs in NFAc-treated slices with no effect on amplitude distribution.

Thus, compromising astroglial function induced a similar reduction in spontaneous release of glutamate to that seen with a co-agonist site antagonist, or enzymatic degradation of D-serine with RgDAAO, suggesting that astroglial release provides the D-serine required for tonic activation of the preNMDAr. However, the effect of incubation with NFAc was somewhat less than that of RgDAAO (cf. Fig. 4) and we cannot exclude the possible influence of neuronal D-serine at the preNMDAR.

We next examined the ability of exogenous D-serine to rescue the reduction in mEPSC frequency induced by preincubation with NFAc in five slices. In 5 neurones the mean frequency of mEPSCs was again lower than that seen in naïve slices and a significant recovery was seen upon addition of D-serine (100 $\mu \mathrm{M}$; Fig. 5B), suggesting that it was indeed the loss of an endogenous D-serine supply from astroglia that resulted in a decline of tonic facilitation.

We also determined whether a loss of endogenous D-serine would reduce the ability of direct activation of preNMDAr to facilitate glutamate release. To this end we compared the ability of NMDA to increase mEPSC frequency in the presence or absence of NFAc. In control neurones $(n=5)$, application of NMDA $(100 \mu M)$ elicited an increase in frequency of mEPSCs of $131 \%$ (from $0.56 \pm 0.17 \mathrm{~Hz}$ to $1.03 \pm 0.25 \mathrm{~Hz}$ ) with no change in amplitude $(7.4 \pm$ $0.5 \mathrm{pA} \vee 7.4 \pm 0.3 \mathrm{pA}$ ). In 5 matched neurones treated with NFAc, the increase in frequency seen with NMDA was only $58 \%(0.32 \pm 0.08 \mathrm{~Hz} \vee 0.49 \pm 0.12 \mathrm{~Hz})$, again without change in amplitude $(7.5 \pm 0.5 \mathrm{pA} \vee 7.2 \pm 0.6 \mathrm{pA})$ suggesting that a loss of endogenous D-serine from astroglial cells could compromise the ability of exogenous NMDA to elevate glutamate release.

\subsection{Effects of UBP 141, purinergic receptor blockade and mGluR antagonists}

Thus, the most parsimonious explanation of our data is that D-serine released from astrocytes is likely to be the coagonist at the preNMDAr on nerve terminals. However, there are a number of other possible, and more complex, interpretations. and we have attempted to rule some of these out with pharmacological approaches.

Astrocytes themselves express NMDAr, and it has been suggested that these are involved in neurone-to-glia signalling and vice versa (Lalo et al., 2006 and Palygin et al., 2011). If these NMDAr responded to ambient glutamate, this could result in tonic release of astrocytic glutamate or other gliotransmitters (e.g. purines). This could 
complicate our interpretations, since treatments aimed to modify the co-agonist site could target the astrocyte NMDAr rather than the glutamate terminal receptors. We have shown that the preNMDAr in the EC is likely to be principally, if not exclusively, a GluN1-GluN2B heteromer (Woodhall et al., 2001, Yang et al., 2006 and Chamberlain et al., 2008). Recent studies have suggested that NMDAr on cortical astrocytes may be largely GluN2C/D-N3containing heteromers (Palygin et al., 2011). We tested the effects of the relatively specific GluN2C/D antagonist, UBP 141 (2.5 $\mu \mathrm{M}$; Morley et al., 2005), alone and in combination with DCKA. The mean frequency of mEPSCs in control conditions $(n=4)$ was $0.43 \pm 0.09 \mathrm{~Hz}$ and this was unaltered by UBP $141(0.45 \pm 0.08 \mathrm{~Hz})$, but subsequently reduced by DCKA $(0.21 \pm 0.02 \mathrm{~Hz})$ in the continued presence of the antagonist. This argues against the reduction in tonic glutamate release induced by the co-agonist blocker occurring via an interaction with NMDAr on astrocytes.

Notwithstanding the failure of UBP 141 to alter the release of glutamate or the effects of DCKA, we have considered other further scenarios dependent upon NMDAr activation of astrocytes by ambient glutamate. One possibility could involve a complex cascade mediated by purinergic transmitters. NMDAr activation can release ATP from astrocytes, and also lead, directly via release or indirectly via metabolism of ATP, to accumulation of extracellular adenosine (e.g. see Queiroz et al., 1997, Pascual et al., 2005 and Wall and Dale, 2013). Subsequent activation of purinergic receptors on neurones or glia could then potentially amplify tonic release of glutamate. Thus, the decrease in glutamate release seen with DCKA or scavenging of D-serine could result not from compromising preNMDAr but, rather, affecting those on the glial cells. We felt that this was unlikely on the basis of the UBP 141 experiments, but also because we have previously shown that both ATP and adenosine reduce, rather than enhance glutamate release in the EC (Saunders, J. and Jones R.S.G., unpublished). However, since the potential mechanism would depend on tonic activation of glutamate release by the astrocytic derived purines, we examined its viability by testing DCKA in the presence of broad spectrum purinergic receptor antagonists; suramin, which non-specifically targets P2 receptors, and CGS15943, which blocks $A_{1}, A_{2 A}, A_{2 B}$ and $A_{3}$ adenosine receptors. Neither frequency $(0.50 \pm 0.07 \vee 0.45$ $\pm 0.04 \mathrm{~Hz})$ nor amplitude $(9.6 \pm 1.9 \vee 9.3 \pm 1.8 \mathrm{pA})$ of mEPSCs was altered by suramin $(100 \mu \mathrm{M}, \mathrm{n}=4)$. Subsequent perfusion with DCKA, in the continued presence of suramin, elicited a similar reduction in frequency (to $0.24 \pm 0.02$ $\mathrm{Hz}$ ) to that seen with the co-agonist site blocker alone (Section 3.2), again without change in amplitude (8.2 \pm 1.4 pA). Likewise, studies with CGS15943 $(1 \mu \mathrm{M}, \mathrm{n}=4)$ showed no change in frequency with the adenosine antagonist alone $(0.30 \pm 0.22 \vee 0.29 \pm 0.07 \mathrm{~Hz})$, but a significant decrease with subsequent addition of DCKA $(0.19 \pm 0.04 \mathrm{~Hz})$.

One further alternative mechanism could involve activation of metabotropic glutamate receptors (mGluR). Glutamate, released from astrocytes in response to NMDAr activation, has been shown to further tonically increase neuronal glutamate release via mGluRs (e.g. see Fiacco and McCarthy, 2004 and Clasadonte and Haydon, 2012). If such a situation was operative at EC synapses, then negatively modifying the co-agonist activation of astrocytic NMDAr could indirectly manifest itself as a reduced frequency of mEPSCs. We have shown previously that agonists at group III (Evans et al., 2000), group II (Morgan, N. et al., unpublished) and group I mGluR (Yang, J. et al., unpublished) agonists can all (under some circumstances) facilitate spontaneous release of glutamate in layer $\mathrm{V}$ of the EC, so such a scenario is possible. However, again for this scenario to be viable, the mGluRs in question would have to be tonically activated. However, we have also shown that the group III receptor antagonist, CPPG does not affect mEPSC frequency (Evans et al., 2000). We now report here that mEPSC frequency is unaltered $(0.66 \pm 0.10 \mathrm{v} 0.71 \pm 0.09 \mathrm{~Hz})$ by the group II mGluR antagonist, LY341495 $(20 \mathrm{nM}, \mathrm{n}=7)$ or by the group I/II antagonist, MCPG (300 $\mu \mathrm{M} ; 0.81 \pm$ $0.11 \vee 0.75 \pm 1.0 \mathrm{~Hz} ; \mathrm{n}=5$ ). Neither the group III or the group I/II antagonist altered mEPSC amplitude (not shown) although there was a slight but significant increase in amplitude with LY341495 (12.3 \pm 0.2 pA v $14.1 \pm 0.2$ pA; P< $0.05)$. Overall these results suggest that mGluR activation following astroglial glutamate release is unlikely to be a complicating factor in the current study.

\section{Discussion}

The obligatory binding of a co-agonist amino acid to a binding site on the NMDAr for its activation was demonstrated 25 years ago (Johnson and Ascher, 1987, Forsythe et al., 1988 and Kleckner and Dingledine, 1988). Enhancement of NMDAr-mediated responses by glycine initially suggested that this amino acid was the endogenous co-agonist (Johnson and Ascher, 1987, Berger et al., 1998, Bergeron et al., 1998, Forsythe et al., 1988 and Kleckner and 
Dingledine, 1988) and, accordingly, the nomenclature "glycine-binding site" received common usage. However, increasing evidence has pointed to D-serine as an alternative endogenous co-agonist (Mothet et al., 2000, Yang et al., 2003, Basu et al., 2009 and Henneberger et al., 2010; Fossat et al., 2012), although a recent study (Papouin et al., 2012) suggests that synaptic (GluN2A-containing) and extrasynaptic (GluN2B-containing) NMDAr may be differentially gated by D-serine and glycine, respectively (see below). All these studies relate to postNMDArs, and coagonist gating of preNMDArs has received much less attention.

PreNMDArs facilitate the release of glutamate at cortical synapses (Berretta and Jones, 1996a, Woodhall et al., 2001, Sjostrom et al., 2003 and Li and Han, 2007Li et al., 2008). This effect is tonically expressed and dependent on ambient glutamate, but, of necessity, it will also require tonic activation of the obligatory co-agonist binding site. We have studied the basis of a co-agonist regulation of glutamate release at synapses in layer $\mathrm{V}$ of the EC. Neither glycine nor D-serine had any substantial effect on frequency, amplitude or kinetics of AMPA receptor-mediated SEPSCs, although cumulative probability analysis of inter-event interval did indicate a slight increase in frequency with $D$-serine but not glycine. This could support a preference for D-serine as the endogenous co-agonist, but overall the data suggest that co-agonist binding site is saturated, or close to it, under baseline conditions in our slices. The endogenous occupation of the preNMDAr co-agonist binding site was confirmed by application of a competitive antagonist, DCKA, which clearly decreased the frequency of EPSCs. The occlusion of this effect by prior application of 2-AP5 indicated that DCKA was acting at the co-agonist site on the preNMDAr. Thus, at these synapses, the levels of ambient glutamate and its co-agonist at the preNMDAr can modulate the control and maintenance of spontaneous excitation. Li and Han (2007) conducted similar studies in rat visual cortex. They also found little effect of exogenous glycine or D-serine, but noted a decrease in probability of release with a co-agonist binding site blocker, although all their recordings were done in Mg-free conditions so may not be directly comparable.

Using specific scavenging enzymes for glycine and D-serine, we have now shown that the endogenous co-agonist occupying the preNMDAr in the EC is almost certainly D-serine rather than glycine. Previous studies have strongly suggested that D-serine acts as the endogenous co-agonist at NMDArs at many postsynaptic sites (Mothet et al., 2000, Yang et al., 2003, Basu et al., 2009 and Henneberger et al., 2010; Fossat et al., 2012), and that glycine uptake transporters are powerful enough to maintain extracellular glycine below its affinity for the NMDAr co-agonist site (Fossat et al., 2012; Papouin et al., 2012). Interestingly, Papouin et al. (2012) have recently suggested that postNMDArs, located subsynaptically in CA1, are preferentially gated by D-serine, whilst those at extrasynaptic sites preferentially bind glycine. Further, they suggest that this is related to the fact that subsynaptic receptors are principally GluN2A heterodimers which have a higher affinity for D-serine (Priestley et al., 1995 and Madry et al., 2007), whereas extrasynaptically they are GluN2B-containing, which have a higher affinity for glycine (Priestley et al., 1995, Wafford et al., 1995 and Madry et al., 2007). We have shown (Woodhall et al., 2001, Yang et al., 2006 and Chamberlain et al., 2008) that the preNMDAr in the EC has a diheterodimeric GluN1-GluN2B subunit composition (likewise in the visual cortex; Li et al., 2008). It might be expected therefore that they would preferentially bind, and be gated by, glycine rather than D-serine, but this was clearly not the case. Thus, the preNMDARs may represent a pharmacologically distinct population of NMDAr that are bound by endogenous D-serine whilst lacking GluN2A subunits.

Glutamate released from astrocytes can activate neuronal NMDArs (Parri et al., 2001) and it has been shown that this is associated with an increase in sEPSCs, possibly via a presynaptic mechanism (Araque et al., 1998). Jourdain et al. (2007) confirmed that astrocytic derived glutamate could boost synaptic strength by activating neuronal preNMDArs. We asked what was the source of D-serine that tonically co-activates the preNMDAr? Serine racemase (the enzyme that reversibly converts I- to D-serine) and D-serine have been largely localized to astrocytes in the brain. However, there is evidence that cortical neurones may also be a source of the endogenous amino acid (Wolosker et al., 1999, Kartvelishvily et al., 2006, Martineau et al., 2006, Williams et al., 2006, Miya et al., 2008 and Rosenberg et al., 2010), so there is still debate over whether postNMDArs are activated by D-serine released from astrocytes or neurones (e.g. see Rosenberg et al., 2010 and Fossat et al., 2012). We found that inhibiting metabolic activity in astrocytes with NFAc, had the same effect on glutamate release in the EC as scavenging D-serine with 
RgDAAO, or blocking the co-agonist binding site with DCKA. This strongly suggests that the preNMDAr is gated by astrocytic D-serine in the EC, rather than neuronal D-serine. However, a weak (although insignificant), reduction of glutamate release by DCKA in the presence of NFAc, means we cannot entirely rule out a neuronal contribution. Interestingly, a recent study has shown that $D$-serine release is increased in rat hippocampus by Isoleucine via exchange at the asc-1 (alanine-serine-cysteine transporter-1) and this results in enhanced NMDAr-dependent LTP (Rosenberg et al., 2013). We have previously shown that spontaneous glutamate release in layer $\mathrm{V}$ of the EC is unaltered by I-Isoleucine (Cunningham et al., 2004), which is essentially equieffective with d-Isoleucine as a substrate for asc-1 (Fukasawa et al., 2000: Helboe et al., 2003). As asc-1 is not expressed in astrocytes (Helboe et al., 2003 and Rosenberg et al., 2013), this could taken as support for a glial, rather than neuronal source of endogenous D-serine in the EC.

However, overall, the picture emerges that activation of preNMDAr and the resultant increase in synaptic activation may well depend on interaction between glutamate, of neuronal origin, and D-serine, from astrocytes, at tripartite cortical synapses. We believe that this is the most parsimonious explanation of our results. However, since astrocytes themselves express NMDArs, activation of which can lead to release of glutamate and purinergic transmitters, other more complex scenarios are possible, which could reflect co-agonist regulation of glial, rather than terminal NMDAr. We have used pharmacological approaches to look at these possibilities and believe that we have largely ruled out a contribution of glial NMDAr-dependent activation of ATP, adenosine or mGlu receptors as complicating factors in our studies. Thus, whilst we cannot be $100 \%$ sure, we are, nevertheless, confident that preNMDAr on terminals at layer $\mathrm{V}$ synapses are co-activated by neuronal glutamate and astrocytic $\mathrm{D}$-serine.

What are the implications of the tonic co-agonist regulation of preNMDAr for synaptic function in the EC? One interesting possibility is in control of presynaptic receptor trafficking. We have previously shown (Yang et al., 2008) that preNMDArs can diffuse laterally within the presynaptic membrane moving between sites proximal and distal to the active release sites, providing a basis for an activity-dependent tonic regulation of glutamate release and hence synaptic strength. Recent work has suggested that GluN2B-containing postNMDAr have their lateral mobility restricted by D-serine (Papouin et al., 2012). We do not know yet whether the co-agonist binding of D-serine similarly slows diffusion of the preNMDAr, but such an effect, mediated by astroglial activity, could provide a basis for an activity-dependent metaplasticity of transmission at excitatory synapses in the EC.

Interestingly, reactive astrocytosis is a feature of human temporal lobe epilepsy and animal models of the chronic condition (Borges et al., 2003, Borges et al., 2006, Cohen-Gadol et al., 2004, Eid et al., 2008, Shapiro et al., 2008 and Wetherington et al., 2008). A decrease in glutamine synthase activity in association with this has been suggested to elevate synaptic levels of glutamate (see Clasadonte and Haydon, 2012). Furthermore, increased levels of D-serine and serine racemase have been observed in pilocarpine induced epilepsy (Ryu et al., 2010). We have previously shown an enhanced tonic facilitation of glutamate release of preNMDAr in a similar model of chronic epilepsy (Yang et al., 2006), and human temporal lobe epilepsy may be associated with an increased density of preNMDAr at cortical synapses (Steffens et al., 2005). Thus, in temporal lobe epilepsy, we may have a situation where increased effectiveness of preNMDArs per se may be exacerbated by an elevation of synaptic availability of both co-agonists as a result of reactive gliosis, leading to a pathological rise in cortical network excitability and synchrony.

Pharmacologically targeting the astroglial-preNMDAr synaptic amplifier may, therefore, provide a useful therapeutic approach to epilepsy. Use of NMDAr co-agonist binding site blockers has already received consideration (Chiamulera et al., 1990, Singh et al., 1990, Peterson, 1991, Peeters and Vanderheyden, 1992 and Wu et al., 2002). Interestingly, we have previously demonstrated that felbamate can reduce glutamate release in the EC via an action at preNMDArs (Yang et al., 2007), and the drug may act, at least in part, by blocking the glycine/D-serine co-agonist binding site (White et al., 1995, Kuo et al., 2004 and Chang and Kuo, 2007). The co-agonist binding site partial agonist, cycloserine, has anticonvulsant activity (Chung et al., 1984, Baran et al., 1994 and De Sarro et al., 2000; see also Ghasemi and Schachter, 2011), and acts as an antagonist only when a high degree of agonist occupancy of the site is present (Henderson et al., 1990). We have shown here that the preNMDAr co-agonist binding site in the EC is likely 
to be endogenously saturated so this approach may be useful in achieving a degree of selectivity for blocking the receptor. The pro-drug, 4-chlorokynurenine, which is largely inactive but undergoes astrocytic conversion to the coagonist binding site blocker, 7-chlorokynuerenic acid (Zhang et al., 2005), could also be an alternative approach to epilepsy therapy. Finally, inhibition of serine racemase (Panizzutti et al., 2001) or enzymatic metabolism of D-serine (Sacchi et al., 2012) could also provide promising strategies for the development of treatments for epilepsy.

\section{Acknowledgements}

We thank the MRC for a doctoral training award to support Alex Lench, and the University of Bath for provision of laboratory and technical facilities.

\section{References}

M. Andersson, F. Blomstrand, E. Hanse. Astrocytes play a critical role in transient heterosynaptic depression in the rat hippocampal CA1 region. J. Physiol., 585 (2007), pp. 843-852.

A. Araque, R.P. Sanzgiri, V. Parpura, P.G. Haydon. Calcium elevation in astrocytes causes an NMDA receptor-dependent increase in the frequency of miniature synaptic currents in cultured hippocampal neurons. J. Neurosci., 18 (1998), pp. 6822-6829.

H. Baran, W. Löscher, M. Mevissen. The glycine/NMDA receptor partial agonist D-cycloserine blocks kainate-induced seizures in rats. Comparison with MK-801 and diazepam. Brain Res., 652 (1994), pp. 195-200.

A.C. Basu, G.E. Tsai, C.L. Ma, J.T. Ehmsen, A.K. Mustafa, L. Han, Z.I. Jiang, M.A. Benneyworth, M.P. Froimowitz, N. Lange, S.H. Snyder, R. Bergeron, J.T. Coyle. Targeted disruption of serine racemase affects glutamatergic neurotransmission and behavior. Mol. Psychiatry, 14 (2009), pp. 719-727.

V.A. Bender, K.J. Bender, D.J. Brasier, D.E. Feldman. Two coincidence detectors for spike timing-dependent plasticity in somatosensory cortex. J. Neurosci., 26 (2006), pp. 4166-4177.

A.J. Berger, S. Dieudonné, P. Ascher. Glycine uptake governs glycine site occupancy at NMDA receptors of excitatory synapses. J. Neurophysiol., 80 (1998), pp. 3336-3340.

R. Bergeron, T.M. Meyer, J.T. Coyle, R.W. Greene. Modulation of N-methyl-D-aspartate receptor function by glycine transport. P.N.A.S., 95 (1998), pp. 15730-15734.

N. Berretta, R.S.G. Jones. Tonic facilitation of glutamate release by presynaptic NMDA autoreceptors in layer II of the entorhinal cortex. Neuroscience, 79 (1996), pp. 339-344.

N. Berretta, R.S.G. Jones. A comparison of spontaneous synaptic EPSCs in layer V and layer II neurones in the rat entorhinal cortex in vitro. J. Neurophysiol., 76 (1996), pp. 1089-1100.

K. Borges, M. Gearing, D.L. McDermott, A.B. Smith, A.G. Almonte, B.H. Wainer, R. Dingledine. Neuronal and glial pathological changes during epileptogenesis in the mouse pilocarpine model. Exp. Neurol., 182 (2003), pp. 21-34.

K. Borges, D. McDermott, H. Irier, Y. Smith, R. Dingledine. Degeneration and proliferation of astrocytes in the mouse dentate gyrus after pilocarpine-induced status epilepticus. Exp. Neurol., 201 (2006), pp. 416-427.

D.J. Brasier, D.E. Feldman. Synapse-specific expression of functional presynaptic NMDA receptors in rat somatosensory cortex. J. Neurosci., 28 (2008), pp. 2199-2211.

S.E.L. Chamberlain, J. Yang, R.S.G. Jones. The role of NMDA receptor subtypes in short term plasticity in the rat entorhinal cortex. Neural Plast. (2008) http://www.hindawi.com/journals/np/2008/872456/. Chang and Kuo, 2007. H.R. Chang, C.C. Kuo. Characterization of the gating conformational changes in the felbamate binding site in NMDA channels. Biophys. J., 93 (2007), pp. 456-466.

C. Chiamulera, S. Costa, A. Reggiani. Effect of NMDA- and strychnine-insensitive glycine site antagonists on NMDA-mediated convulsions and learning. Psychopharmacology, 102 (1990), pp. 551-552.

S.H. Chung, M.S. Johnson, A.M. Gronenborn. L-cycloserine: a potent anticonvulsant. Epilepsia, 25 (1984), pp. $353-362$.

J. Clasadonte, P.G. Haydon. Astrocytes and epilepsy. J.L. Noebels, M. Avoli, M.A. Rogawski, R.W. Olsen, A.V. Delgado-Escueta (Eds.), Jasper's Basic Mechanisms of the Epilepsies (fourth ed.) (2012) Bethesda (MD) http://www.ncbi.nlm.nih.gov/books/NBK98159/

A.A. Cohen-Gadol, J.W. Pan, J.H. Kim, D.D. Spencer, H.H. Hetherington. Mesial temporal lobe epilepsy: a proton magnetic resonance spectroscopy study and a histopathological analysis. J. Neurosurg., 101 (2004), pp. 613-620. 
R. Corlew, Y. Wang, H. Ghermazien, A. Erisir, B.D. Philpot. Developmental switch in the contribution of presynaptic and postsynaptic NMDA receptors to long-term depression. J. Neurosci., 27 (2007), pp. 9835-9845.

M.O. Cunningham, G.L. Woodhall, S.E. Thompson, D.J. Dooley, R.S.G. Jones. Dual effects of gabapentin and pregabalin on glutamate release at rat entorhinal synapses in vitro. Eur. J. Neurosci., 20 (2004), pp. 1566-1576.

G. De Sarro, S. Gratteri, F. Naccari, M.P. Pasculli, A. De Sarro. Influence of D-cycloserine on the anticonvulsant activity of some antiepileptic drugs against audiogenic seizures in DBA/2 mice. Epilepsy Res., 40 (2000), pp. 109-121.

I.C. Duguid, T.G. Smart. Presynaptic NMDA receptors. A.M. Van Dongen (Ed.), Biology of the NMDA Receptor, CRC Press, Boca Raton (FL) (2009) Chapter 14. PMID: 21204409.

H. Eichenbaum. The long and winding road to memory consolidation. Nat. Neurosci., 4 (2001), pp. 1057-1058.

T. Eid, A. Williamson, T.S. Lee, O.A. Petroff, N.C. de Lanerolle. Glutamate and astrocytes-key players in human mesial temporal lobe epilepsy?. Epilepsia, 49 (Suppl. 2) (2008), pp. 42-52.

D.I. Evans, R.S.G. Jones, G. Woodhall. Activation of presynaptic group III metabotropic receptors enhances glutamate release in rat entorhinal cortex. J. Neurophysiol., 83 (2000), pp. 2519-2525.

S. Fantinato, L. Pollegioni, M.S. Pilone. Engineering, expression and purification of a His-tagged chimeric D-amino acid oxidase from Rhodotorula gracilis. Enzyme Microb. Technol., 29 (2001), pp. 407-412.

T.A. Fiacco, K.D. McCarthy. Intracellular astrocyte calcium waves in situ increase the frequency of spontaneous AMPA receptor currents in ca1 pyramidal neurons. J. Neurosci., 24 (2004), pp. 722-732.

I.D. Forsythe, G.L. Westbrook, M.L. Mayer. Modulation of excitatory synaptic transmission by glycine and zinc in cultures of mouse hippocampal neurons. J. Neurosci., 8 (1988), pp. 3733-3741.

P. Fossat, F.R. Turpin, S. Sacchi, J. Dulong, T. Shi, J.M. Rivet, J.V. Sweedler, L. Pollegioni, M.J. Millan, S.H. Oliet, J.P. Mothet. Glial D-serine gates NMDA receptors at excitatory synapses in prefrontal cortex. Cereb. Cortex, 22 (2012), pp. 595-606.

Y. Fukasawa, H. Segawa, J.Y. Kim, A. Chairoungdua, D.K. Kim, H. Matsuo, S. Cha, H. Endou, Y. Kanai. Identification and characterization of a Naindependent neutral amino acid transporter that associates with the $4 \mathrm{~F} 2$ heavy chain and exhibits substrate selectivity for small neutral $\mathrm{D}$ - and L-amino acids. J. Biol. Chem., 275 (2000), pp. 9690-9698.

M. Ghasemi, S.C. Schachter. The NMDA receptor complex as a therapeutic target in epilepsy: a review. Epilepsy Behav., 22 (2011), pp. 617640 .

B. Hassel, H. Bachelard, P. Jones, F. Fonnum, U. Sonnewald. Trafficking of amino acids between neurons and glia in vivo. Effects of inhibition of glial metabolism by fluoroacetate. J. Cereb. Blood. Flow. Metab., 17 (1997), pp. 1230-1238.

L. Helboe, J. Egebjerg, M. Møller, C. Thomsen. Distribution and pharmacology of alanine-serine-cysteine transporter 1 (asc-1) in rodent brain. Eur. J. Neurosci., 18 (2003), pp. 2227-2238.

G. Henderson, J.W. Johnson, P. Ascher. Competitive antagonists and partial agonists at the glycine modulatory site of the mouse N-methyl-Daspartate receptor. J. Physiol., 430 (1990), pp. 189-212.

C. Henneberger, T. Papouin, S.H. Oliet, D.A. Rusakov. Long-term potentiation depends on release of D-serine from astrocytes. Nature, 463 (2010), pp. 232-236.

Y. Humeau, H. Shaban, S. Bissiere, A. Luthi. Presynaptic induction of heterosynaptic associative plasticity in the mammalian brain. Nature, 426 (2003), pp. 841-845.

S. Hulsmann, H. Straub, D.W. Richter, E.J. Speckmann. Blockade of astrocyte metabolism causes delayed excitation as revealed by voltage sensitive dyes in mouse brainstem slices. Exp. Brain Res., 150 (2003), pp. 117-121.

V. Job, G.L. Marcone, M.S. Pilone, L. Pollegioni. Glycine oxidase from Bacillus subtilis. Characterization of a new flavoprotein. J. Biol. Chem., 277 (2002), pp. 6985-6993.

R.S.G. Jones, G.L. Woodhall. Background synaptic activity in rat entorhinal cortical neurones: differential control of transmitter release by presynaptic receptors. J. Physiol., 562 (2005), pp. 107-120. 
R.S.G. Jones, U. Heinemann. Synaptic and intrinsic responses of medial entorhinal cortical cells in normal and magnesium-free medium in vitro. J. Neurophysiol., 59 (1988), pp. 1476-1496.

J.W. Johnson, P. Ascher. Glycine potentiates the NMDA response in cultured mouse brain neurons. Nature, 325 (1987), pp. 529-531.

P. Jourdain, L.H. Bergersen, K. Bhaukaurally, P. Bezzi, M. Santello, M. Domercq, C. Matute, F. Tonello, V. Gundersen, A. Volterra. Glutamate exocytosis from astrocytes controls synaptic strength. Nat. Neurosci., 10 (2007), pp. 331-339.

E. Kartvelishvily, M. Shleper, L. Balan, E. Dumin, H. Wolosker. Neuron-derived D-serine release provides a novel means to activate N-methyl-Daspartate receptors. J. Biol. Chem., 281 (2006), pp. 14151-14162.

N.W. Kleckner, R. Dingledine. Requirement for glycine in activation of NMDA-receptors expressed in Xenopus oocytes. Science, 241 (1988), pp. 835-837.

C.C. Kuo, B.J. Lin, H.R. Chang, C.P. Hsieh. Use-dependent inhibition of the N-methyl-D-aspartate currents by felbamate: a gating modifier with selective binding to the desensitized channels. Mol. Pharmacol., 65 (2004), pp. 370-380.

U. Lalo, Y. Pankratov, F. Kirchhoff, R.A. North, A. Verkhratsky. NMDA receptors mediate neuron-to-glia signaling in mouse cortical astrocytes. J. Neurosci., 26 (2006), pp. 2673-2683.

R.S. Larsen, R.J. Corlew, M.A. Henson, A.C. Roberts, M. Mishina, M. Watanabe, S.A. Lipton, N. Nakanishi, I. Pérez-Otaño, R.J. Weinberg, B.D. Philpot. NR3A-containing NMDARs promote neurotransmitter release and spike timing-dependent plasticity. Nat. Neurosci., 14 (2011), pp. 338-344.

Y.H. Li, T.Z. Han. Glycine binding sites of presynaptic NMDA receptors may tonically regulate glutamate release in the rat visual cortex. J. Neurophysiol., 97 (2007), pp. 817-823.

Y.H. Li, T.Z. Han, K. Meng. Tonic facilitation of glutamate release by glycine binding sites on presynaptic NR2B-containing NMDA autoreceptors in the rat visual cortex. Neurosci. Lett., 432 (2008), pp. 212-216.

C. Madry, I. Mesic, H. Betz, B. Laube. The N-terminal domains of both NR1 and NR2 subunits determine allosteric $\mathrm{Zn}^{2+}$ inhibition and glycine affinity of $\mathrm{N}$-methyl-D-aspartate receptors.

M. Martineau, G. Baux, J.P. Mothet. D-serine signalling in the brain: friend and foe. Trends Neurosci., 29 (2006), pp. $481-491$.

R.M. Morley, H.W. Tse, B. Feng, J.C. Miller, D.T. Monaghan, D.E. Jane. Synthesis and pharmacology of N1-substituted piperazine-2,3dicarboxylic acid derivatives acting as NMDA receptor antagonists, J. Med. Chem., 48 (2005), pp. 2627-2637.

J.P. Mothet, A.T. Parent, H. Wolosker, R.O. Brady, D.J. Linden, C.D. Ferris, M.A. Rogawski, S.H. Snyder. D-serine is an endogenous ligand for the glycine site of the N-methyl-D-aspartate receptor. P.N.A.S., 97 (2000), pp. 4926-4931.

K. Miya, R. Inoue, Y. Takata, M. Abe, R. Natsume, K. Sakimura, K. Hongou, T. Miyawaki, H. Mori. Serine racemase is predominantly localized in neurons in mouse brain. J. Comp. Neurol., 510 (2008), pp. 641-654.

A. Okada-Ogawa, I. Suzuki, B.J. Sessle, C.Y. Chiang, M.W. Salter, J.O. Dostrovsky, Y. Tsuboi, M. Kondo, J. Kitagawa, A. Kobayashi, N. Noma, Y. Imamura, K. Iwata. Astroglia in medullary dorsal horn (trigeminal spinal subnucleus caudalis) are involved in trigeminal neuropathic pain mechanisms. J. Neurosci., 29 (2009), pp. 11161-11171.

O. Palygin, U. Lalo, Y. Pankratov. Distinct pharmacological and functional properties of NMDA receptors in mouse cortical astrocytes. Br. J. Pharmacol., 163 (2011), pp. 1755-1766.

R. Panizzutti, J. De Miranda, C.S. Ribeiro, S. Engelender, H. Wolosker. A new strategy to decrease N-methyl-D-aspartate (NMDA) receptor coactivation: inhibition of D-serine synthesis by converting serine racemase into an eliminase. P.N.A.S., 98 (2001), pp. 5294-5299.

T. Papouin, L. Ladépêche, J. Ruel, S. Sacchi, M. Labasque, M. Hanini, L. Groc, L. Pollegioni, J.P. Mothet, S.H. Oliet. Synaptic and extrasynaptic NMDA receptors are gated by different endogenous co-agonists. Cell, 150 (2012), pp. 633-646.

H.R. Parri, T.M. Gould, V. Crunelli. Spontaneous astrocytic $\mathrm{Ca}^{2+}$ oscillations in situ drive NMDAR-mediated neuronal excitation. Nat. Neurosci., 4 (2001), pp. 803-812.

O. Pascual, K.B. Casper, C. Kubera, J. Zhang, R. Revilla-Sanchez, J.Y. Sul, H. Takano, S.J. Moss, K. McCarthy, P.G. Haydon. Astrocytic purinergic signalling coordinates synaptic networks. Science, 310 (2005), pp. 113-116. 
B.W. Peeters, P.M. Vanderheyden. In vitro and in vivo characterization of the NMDA receptor-linked strychnine-insensitive glycine site. Epilepsy Res., 12 (1992), pp. 157-162.

S.L. Peterson. Anticonvulsant drug potentiation by glycine in maximal electroshock seizures is mimicked by D-serine and antagonized by 7 chlorokynurenic acid. Eur. J. Pharmacol., 199 (1991), pp. 341-348.

T. Priestley, P. Laughton, J. Myers, B. Le Bourdellés, J. Kerby, P.J. Whiting. Pharmacological properties of recombinant human N-methyl-Daspartate receptors comprising NR1a/NR2A and NR1a/NR2B subunit assemblies expressed in permanently transfected mouse fibroblast cells. Mol. Pharmacol., 48 (1995), pp. 841-848.

G. Queiroz, P.J. Gebicke-Haerter, A. Schobert, K. Starke, I. von Kugelgen. Release of ATP from cultured rat astrocytes elicited by glutamate receptor activation. Neuroscience, 78 (1997), pp. 1203-1208.

A. Rodríguez-Moreno, A. Banerjee, O. Paulsen. Presynaptic NMDA receptors and spike timing-dependent depression at cortical synapses. Front. Synaptic Neurosci., 2 (2010), p. 18.

D. Rosenberg, E. Kartvelishvily, M. Shleper, C.M. Klinker, M.T. Bowser, H. Wolosker. Neuronal release of D-serine: a physiological pathway controlling extracellular D-serine concentration. FASEB J., 24 (2010), pp. 2951-2961.

D. Rosenberg, S. Artoul, A.C. Segal, G. Kolodney, I. Radzishevsky, E. Dikopoltsev, V.N. Foltyn, R. Inoue, H. Mori, J.M. Billard, H. Wolosker. Neuronal D-serine and glycine release via the Asc-1 transporter regulates NMDA receptor-dependent synaptic activity. J. Neurosci., 33 (2013), pp. 3533-3544.

H.J. Ryu, J.E. Kim, S.I. Yeo, D.S. Kim, O.S. Kwon, S.Y. Choi, T.C. Kang. Potential roles of D-serine and serine racemase in experimental temporal lobe epilepsy. J. Neurosci. Res., 88 (2010), pp. 2469-2482.

S. Sacchi, L. Caldinelli, P. Cappelletti, L. Pollegioni, G. Molla. Structure-function relationships in human D-amino acid oxidase. Amino Acids, 43 (2012), pp. 1833-1850.

R.D. Samson, D. Pare. Activity-dependent synaptic plasticity in the central nucleus of the amygdala. J. Neurosci., 25 (2005), pp. $1847-1855$.

L.A. Shapiro, L. Wang, C.E. Ribak. Rapid astrocyte and microglial activation following pilocarpine-induced seizures in rats. Epilepsia, 49 (Suppl. 2) (2008), pp. 33-41.

L. Singh, C.A. Vass, J.C. Hunter, G.N. Woodruff, J. Hughes. The anticonvulsant action of Cl-977, a selective kappa-opioid receptor agonist: a possible involvement of the glycine/NMDA receptor complex. Eur. J. Pharmacol., 191 (1990), pp. 477-480.

P.J. Sjostrom, G.G. Turrigiano, S.B. Nelson. Neocortical LTD via coincident activation of presynaptic NMDA and cannabinoid receptors. Neuron, 39 (2003), pp. 641-654.

L.R. Squire, C.E. Stark, R.E. Clark. The medial temporal lobe. Ann. Rev. Neurosci., 27 (2004), pp. 279-306.

M. Steffens, H.J. Huppertz, J. Zentner, E. Chauzit, T.J. Feuerstein. Unchanged glutamine synthetase activity and increased NMDA receptor density in epileptic human neocortex: implications for the pathophysiology of epilepsy. Neurochem. Int., 47 (2005), pp. 379-384.

K.A. Wafford, M. Kathoria, C.J. Bain, G. Marshall, B. Le Bourdellès, J.A. Kemp, P.J. Whiting. Identification of amino acids in the N-methyl-Daspartate receptor NR1 subunit that contribute to the glycine binding site. Mol. Pharmacol., 47 (1995), pp. 374-380.

M.J. Wall, N. Dale. Neuronal transporter and astrocytic ATP exocytosis underlie activity-dependent adenosine release in the hippocampus. J. Physiol., 591 (2013), pp. 3853-3871.

J. Wetherington, G. Serrano, R. Dingledine. Astrocytes in the epileptic brain. Neuron, 58 (2008), pp. 168-178.

H.S. White, W.L. Harmsworth, R.D. Sofia, H.H. Wolf. Felbamate modulates the strychnine-insensitive glycine receptor. Epilepsy Res., 20 (1995), pp. 41-48.

S.M. Williams, C.M. Diaz, L.T. Macnab, R.K. Sullivan, D.V. Pow. Immunocytochemical analysis of D-serine distribution in the mammalian brain reveals novel anatomical compartmentalizations in glia and neurons. Glia, 53 (2006), pp. 401-411.

M.P. Witter, E.I. Moser. Spatial representation and the architecture of the entorhinal cortex. Trends Neurosci., 29 (2006), pp. 671-678.

H. Wolosker, S. Blackshaw, S.H. Snyder. Serine racemase: a glial enzyme synthesizing D-serine to regulate glutamate-N-methyl-D-aspartate neurotransmission. P.N.A.S., 96 (1999), pp. 13409-13414. 
G.L. Woodhall, D.I.P. Evans, M.O. Cunningham, R.S.G. Jones. NR2B-containing NMDA autoreceptors at synapses on entorhinal cortical neurones. J. Neurophysiol., 86 (2001), pp. 1644-1651.

G.L. Woodhall, S.J. Bailey, S.E. Thompson, D.I. Evans, R.S.G. Jones. Fundamental differences in spontaneous synaptic inhibition between deep and superficial layers of the rat entorhinal cortex. Hippocampus, 15 (2005), pp. 232-245.

H.Q. Wu, S.C. Lee, H.E. Scharfman, R. Schwarcz. L-4-chlorokynurenine attenuates kainate-induced seizures and lesions in the rat. Exp. Neurol., 177 (2002), pp. 222-232.

Y. Yang, W. Ge, Y. Chen, Z. Zhang, W. Shen, C. Wu, M. Poo, S. Duan. Contribution of astrocytes to hippocampal long-term potentiation through release of D-serine. P.N.A.S., 100 (2003), pp. 15194-15199.

J. Yang, S.E.L. Chamberlain, G.L. Woodhall, R.S.G. Jones. Mobility of NMDA autoreceptors but not postsynaptic receptors in the rat entorhinal cortex. J. Physiol., 586 (2008), pp. 4905-4924.

J. Yang, C. Wetterstrand, R.S.G. Jones. Felbamate but not phenytoin or gabapentin reduces glutamate release by blocking presynaptic NMDA receptors in the entorhinal cortex. Epilepsy Res., 77 (2007), pp. 157-164.

J. Yang, G.L. Woodhall, R.S.G. Jones. Tonic facilitation of glutamate release by presynaptic NR2B-containing NMDA receptors is increased in the entorhinal cortex of chronically epileptic rats. J. Neurosci., 26 (2006), pp. 406-410.

D.X. Zhang, J.M. Williamson, H.Q. Wu, R. Schwarcz, E.H. Bertram. In situ-produced 7-chlorokynurenate has different effects on evoked responses in rats with limbic epilepsy in comparison to naive controls. Epilepsia, 46 (2005), pp. 1708-1715. 